\title{
New Strategy for Analog Circuit Performance Evaluation under Disturbance and Fault Value
}

\author{
Aihua Zhang, ${ }^{1}$ Yongchao Wang, ${ }^{1}$ Chen Chen, ${ }^{1}$ and Hamid Reza Karimi ${ }^{2}$ \\ ${ }^{1}$ College of Engineering, Bohai University, Jinzhou 121013, China \\ ${ }^{2}$ Department of Engineering, Faculty of Engineering and Science, The University of Agder, Grimstad 4898, Norway
}

Correspondence should be addressed to Aihua Zhang; jsxinxi_zah@163.com

Received 23 December 2013; Accepted 6 January 2014; Published 13 February 2014

Academic Editor: Xudong Zhao

Copyright (C) 2014 Aihua Zhang et al. This is an open access article distributed under the Creative Commons Attribution License, which permits unrestricted use, distribution, and reproduction in any medium, provided the original work is properly cited.

\begin{abstract}
Focus on this issue of disturbance and fault value is inevitable in data collection about analog circuit. A novel strategy is developed for analog circuit online performance evaluation based on fuzzy learning and double weighted support vector machine (DWMKFSVM). First, the double weighted support vector regression machine is employed to be the indirect evaluation means, relied on the college analog electronic technology experiment to evaluate analog circuit. Second, the superiority of fuzzy learning also is addressed to realize active suppression to the fault values and disturbance parameters. Moreover, the multikernel RBF is employed by support vector regression machine to realize more flexibility online such as the bandwidths tuning. Numerical results, supported by the college analog circuit experiments, adopted OTL performance eight indexes, which were obtained via precision instrument evaluation in two years to construct training set and are then to be evaluated online based on DWMK-FSVM. Simulation results presented not only highlight precision of the evaluation strategy derived here but also illustrate its great robustness.
\end{abstract}

\section{Introduction}

Analog circuits are one of the fundamental parts of modern electromechanic systems. Much digital electronic technology has swarmed into our lives; it seems that all the analog circuits are going to decline, but there still exists a need to use analog circuits [1]. Such as analog circuit is still used to convert speech signals to digital signals, sensor signals are inputted to microprocessors, and digital outputs are converted to analog signals. Furthermore, all the digital systems cannot divorce from ultimately analog circuits [2].

The presence of analog circuit detection capability is vital when focusing on the status discussed above. Physical damage, manufacturing faults, aging, radiation, temperature changes, and power surges are possible reasons; therefore the performance evaluation should be done beforehand. If the system has no performance evaluation part, there is a high risk possibility, especially when the analog circuit is on the status of radical performance degradation. Even worse, analog circuits have been widely used for the autonomous systems working without the intervention of human operators in remote and hazardous environments. The purpose of analog circuits performance evaluation is to prevent catastrophic accidents and give a caution to the system. In the previous studies, there have been a lot of works about the automation of the process, especially with evolutionary computation [3-5]. Evolutionary computation includes a set of methodologies mimicking the natural evolution phenomena: neural network [6-9], genetic algorithm [6, 10], fuzzy logic $[11,12]$, switch system [13-16] and data driven [17-20]. Zhang and $\mathrm{Yu}[20]$ attempted to evolve analog circuits performance evaluation strategy using support vector regression based on data information about normal analog circuits such as four indexes, input resistance, input voltage, output resistance, and output voltage. This method has presented some advantages such as well evaluation accuracy, portability, and low cost; however, this design has ignored the reality status that the processing of the data collection includes fault value or disturbance value. The low convergence rate could not meet the online evaluation requirement. Literature [21] focused the singularity issue proposed weighted LSSVM algorithm. Based on the idea discussed in literature [21], fuzzy clustering idea is also drawn into LSSVM which guarantees the robust via weighting for each sample [22]. Although there have been 
several works on declining singularity effect to this evaluation accuracy with weight to obtain the robustness the weighted value is constant which is susceptible to be influenced by the parameter selection. In fact, Kawamura et al. have leaded robust RBF net to robust LSSVR in early years, yet the selection and structure determination of the weight initial value is still the most difficult problem [23].

In this paper, we argue that the evolutionary computation can be useful to realize analog circuit online performance evaluation under disturbance and fault value based on fuzzy learning theory and double weighted support vector machine, and the kernel function of which employs the multikernel RBF. Numerical results, supported by the college analog circuit experiments, adopted OTL performance eight indexes, which were obtained via precision instrument evaluation in two years to construct training set, and are then to be evaluated online based on DWMK-FSVM. Simulation results presented not only highlight speed ability of the evaluation strategy derived here but also illustrate its great precision and robustness.

\section{OTL Technical Index}

The main technical index of OTL includes gain, transmission bands, center frequency, upper/lower cut-off frequency, maximum output extent, as the important technical index, nonlinear distortion coefficient, input/output resistance, and maximum output power. In addition, aiming at different situation, as the other technical index, power capacity, antiinterference ability, signal to noise ratio, weight, volume, working temperature, and so forth may be proposed. During this technical index, gain embodies the amplified ability, transmission bands give out the response ability to the input signal, and upper/lower cut-off frequency gives out the input/output signal frequency band threshold. When the quiescent point of OTL is confirmed, the four technical indexes such as gain, transmission bands, and upper/lower cut-off frequency are the important.

\section{Analog Circuit Performance Evaluation Strategy}

3.1. Fuzzy Support Vector Machine (FSVM). The development of SVM came from the best optimum hyperplane which has the linearly separable property. For one training set with classification markers, $\left(x_{i}, y_{i}\right), x_{i} \in R^{n}$ and $y_{i} \in\{+1,-1\}, i=$ $1, \ldots, l$, if hyperplane $\widetilde{\omega} \cdot x+b=0$ could divide the samples into two types correctly; the best optimum hyperplane would make the sums of the two types to the hyperplane distance be the maximum. The best optimum hyperplane may be obtained via solving the following optimization problem:

$$
\begin{array}{ll}
\min & \frac{1}{2}\|\widetilde{\omega}\|+C \sum_{i=1}^{l} \xi_{i} \\
\text { s.t. } & y_{i}\left[\left(\widetilde{\omega} \cdot x_{i}\right)+b\right] \geq 1, \quad \xi_{i} \geq 0, i=1, \ldots, l,
\end{array}
$$

where $\xi_{i}$ is error expression and $C$ is penalty factor.
Tthe solving problem of best optimum hyperplane above may be transformed into dual problem via utilizing Lagrange multiplier method.

Consider

$$
\begin{array}{ll}
\max & \sum_{i=1}^{l} a_{i}-\frac{1}{2} \sum_{i, j=1}^{l} a_{i} a_{j} y_{i} y_{j}\left(x_{i} \cdot x_{j}\right) \\
\text { s.t. } & \sum_{i=1}^{l} a_{i} y_{i}=0, \quad 0 \leq a_{i} \leq C, \quad i=1, \ldots, l,
\end{array}
$$

where $a_{i}$ is the Lagrange multiplier of $x_{i}$. Equation (2) is a typical optimal problem of quadratic function, therefore there will be a guarantee for the existence of a unique solution.That is $a_{i} \neq 0$ and the corresponding samples are support vector. So the decision function should be

$$
f(x)=\operatorname{sign}\left(\sum_{i=1}^{l} a_{i} y_{i}\left(x_{i} \cdot x\right)+b\right) .
$$

For the nonseparable problem, we can solved this issue via high dimensional mapping $\phi: R^{n} \rightarrow H$, and the linear classification would be realized in the feature space. By defining the function $k\left(x_{i}, x_{j}\right)=\phi\left(x_{i}\right) \cdot \phi\left(x_{j}\right)$, the dual programming of formula (2) would be

$$
\begin{array}{ll}
\max & \sum_{i=1}^{l} a_{i}-\frac{1}{2} \sum_{i, j=1}^{l} a_{i} a_{j} y_{i} y_{j} k\left(x_{i} \cdot x_{j}\right) \\
\text { s.t. } & \sum_{i=1}^{l} a_{i} y_{i}=0, \quad 0 \leq a_{i} \leq C, \quad i=1, \ldots, l .
\end{array}
$$

The corresponding decision function would be

$$
f(x)=\operatorname{sign}\left(\sum_{i=1}^{l} a_{i} y_{i} k\left(x_{i} \cdot x\right)+b\right),
$$

where $k\left(x_{i}, x_{j}\right)=\phi\left(x_{i}\right) \cdot \phi\left(x_{j}\right)$ is the kernel function; the selection of kernel function should make it to be one of its feature space inner product. The common kernel function includes polynomial kernel function (6); Gauss RBF kernel function (7), and sigmoid kernel function (8), which are expressed as follows:

$$
\begin{gathered}
k\left(x_{i}, x_{j}\right)=\left(x_{i} \cdot x_{j}+1\right)^{d}, \quad d=1,2, \ldots n ; \\
k\left(x_{i}, x_{j}\right)=\exp \left(-\gamma\left\|x_{i}-x_{j}\right\|^{2}\right), \\
k\left(x_{i}, x_{j}\right)=\tanh \left(b\left(x_{i} \cdot x_{j}\right)+c\right), \quad b>0, c<0 .
\end{gathered}
$$

Like SVM, the aim of the FSVM [24] is to find an optimal separating hyperplane that separates the sample points into two classes with the maximal margin. The quadratic optimization problem for classification is considered as the solution to

$$
\begin{array}{ll}
\min & \frac{1}{2}\|\widetilde{\omega}\|+C \sum_{i=1}^{l} s_{i} \xi_{i} \\
\text { s.t. } & y_{i}\left[\left(\widetilde{\omega} \cdot x_{i}\right)+b\right] \geq 1-\xi_{i}, \quad \xi_{i} \geq 0, i=1, \ldots, l .
\end{array}
$$


For considering the effect under the feature strong or weak, the weighted idea has been employed.

3.2. Double Weighted Multikernel FSVM (DWMK-FSVM). Double weighted FSVM (DW-FSVM) is one method that should not only consider the sample number difference and sample importance difference, but also give attention to influence above two characteristics of the feature strong and weak at the same time. DW-FSVM improved the classification precision and conformed FSVM to have better robustness. For illustrative purposes, the DW-FSVM constructed steps $[25,26]$ are presented as follows.

Step 1. Let source dataset be $R$, where $R=\left\{x_{i}, x_{j}\right\}_{i=1}^{j}, x_{i} \in R^{n}$, $x_{j} \in\{-1,+1\}$ and training the FSVMs via $R$.

Step 2. Based on FSVMs' training result, we eliminated the samples and obtained the dataset $S$,

$x_{i}^{+} \in$ positive example, $f\left(x_{i}^{+}\right)>1$ or $f\left(x_{i}^{+}\right)<0$,

$x_{i}^{+} \in$ negative example, $f\left(x_{i}^{+}\right)<-1$ or $f\left(x_{i}^{+}\right)>0$.

Step 3. We eliminated the redundancy samples of $S$, obtained the support vector dataset $P$ which included part redundancy samples:

$$
P=\left\{x_{i}^{+}, 1\right\}_{i=1}^{l^{+}} \cup\left\{y_{j}^{-},-1\right\}_{j=1}^{l^{-}},
$$

where $x_{i} \in R^{n}, l^{+}$is the positive example number of database $P, l^{-}$is the negative example number of database $P, x_{i}^{+}$is the positive example, and $y_{j}^{-}$is the negative example.

Step 4. we calculated the essentiality weight of $P$ via (13), and the sample error variance value, which is needed during the calculation, can be obtained by initial training FSVMs

$$
\begin{aligned}
& m l_{i}^{+}=1-\frac{\xi_{i}^{+}-\min _{i} \xi_{i}^{+}}{\max _{i} \xi_{i}^{+}-\min _{i} \xi_{i}^{+}}+\varepsilon, \\
& m l_{j}^{-}=1-\frac{\xi_{j}^{-}-\min _{j} \xi_{j}^{-}}{\max _{j} \xi_{j}^{-}-\min _{j} \xi_{j}^{-}}+\varepsilon,
\end{aligned}
$$

where $\varepsilon$ is a little positive number and category weight may be obtained via $S^{+} / S^{-}=l^{-} / l^{+}$.

Step 5. The samples of dataset $P$ were described by feature dataset $\{c f, u c\}$, where $c f$ is the class label feature and $u c=$ $\left(u_{1}, u_{2}, \ldots, u_{n}\right)$ is the nonclass label feature set.

Step 6. We calculated feature $u c_{i}(1 \leq i \leq n)$ information gain, constructed feature weight vector $\omega$ and linearity transform diagonal matrix DM:

$$
\begin{gathered}
\omega=\sqrt{G}=\left(\sqrt{\operatorname{Gain}\left(u c_{1}\right)}\right), \ldots, \sqrt{\operatorname{Gain}\left(u c_{n}\right)} \\
\mathrm{DM}=\operatorname{diag}(\omega),
\end{gathered}
$$

where $\mathrm{DM}_{k k^{\prime}}=\omega_{k} \delta_{k k^{\prime}}$.

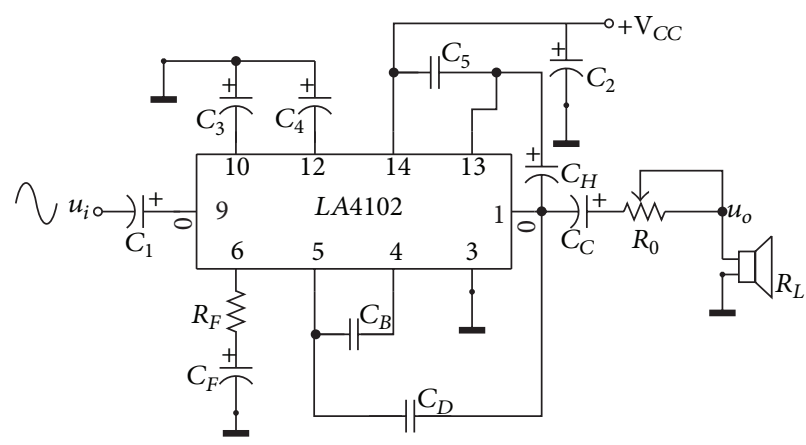

FIgure 1: The OTL Power Amplifier with Bootstrap Circuit.

Step 7. We replaced FSVM kernel function formulas (6)-(8) with feature weight kernel (15) and selected the appropriate model and training arithmetic to construct classification decision function for dataset $P$ as follows

$$
\begin{aligned}
k_{p}\left(x_{i}, x_{j}\right) & =\left(x_{i}^{T} P \cdot x_{j}^{T} P+1\right)^{d} \\
& =\left(x_{i}^{T} P P^{T} x_{j}+1\right)^{d}, \quad d=1,2, \ldots, \\
k_{p}\left(x_{i}, x_{j}\right) & =\exp \left(-\gamma\left\|x_{i}^{T} P-x_{j}^{T} P\right\|^{2}\right) \\
& =\exp \left(-\gamma\left(x_{i}-x_{j}\right)^{T} P P^{T}\left(x_{i}-x_{j}\right)\right), \\
k_{p}\left(x_{i}, x_{j}\right) & =\tanh \left(b\left(x_{i}^{T} P \cdot x_{j}^{T} P\right)+c\right) \\
& =\tanh \left(b\left(x_{i}^{T} P P^{T} x_{j}\right)+c\right), \quad b>0, c<0 .
\end{aligned}
$$

In this paper, the feature weighted Gauss RBF kernel function is employed. And the multikernel idea [27] is also imported in this part which can make itself have more flexibility to the kernel function online such as the bandwidths tuning. And the multikernel RBF is defined by

$$
\operatorname{ker}\left(x_{i}, x_{j}\right)=\sum_{m=1}^{p} \frac{k_{m} \exp \left(-d_{i, j} / 2 \sigma_{m}^{2}\right)}{\sum_{z=1}^{p} k_{z}}
$$

\section{Experiment Results and Analysis}

Experiment based on the test circuit OTL, which is a typical bootstrap circuit shown in Figure 1, and the experiment data, eight indexes, are obtained by precise instrument evaluation in two years. The sample number is $400 \times 100$ which is recorded as dataset $R$. Before verifying the proposed method in this paper, the first thing to be done is establishing datasets of training and testing. However, the disturbance value and fault value in the data set which were caused by industry field influence and other noncircuit fault factors will have great effects on model performance of SVM, especially the dataset, which include the strangeness value, are still used for modeling. Hence, a normalization of the data is required before presenting the input patterns to any statistical machine 
TABLE 1: Result of data feature and comparative experiment of regression problem on experiment data.

\begin{tabular}{lcccccccc}
\hline TRSN & TESN & FN & Method & Parameter $(\sigma, \varepsilon, \gamma)$ & SVN & TRMSE & TEMSE & CPU/s \\
\hline $259 \times 100$ & $59 \times 100$ & 8 & MKALSSVR & $(200,0.1,0.75)$ & 30 & $1.6982 e-014$ & $1.7001 e-028$ & 0.021 \\
$259 \times 100$ & $59 \times 100$ & 8 & LSSVR & $(200,0.1, /)$ & 1376 & $4.5544 e-015$ & $4.2437 e-012$ & 2.981 \\
$259 \times 100$ & $59 \times 100$ & 8 & $\varepsilon$-SVR & $(200,0.1, /)$ & 1607 & $5.4003 e-017$ & $5.3025 e-004$ & 0.062 \\
\hline
\end{tabular}

SVN denotes the number of support vector, TRSN denotes the number of training support vector, TESN denotes the number of testing support vector, FN denotes the number of the data feature, TEMSE denotes the testing data mean square error, and TDMSE denotes the training data mean square error.

learning algorithm. In this experiment, 0-1 normalization method, denoted by (17), is utilized to preprocess.

$$
x_{i}^{n}=\frac{x_{i}^{a}-x_{i}^{\min }}{x_{i}^{\max }-x_{i}^{\min }},
$$

where $x_{i}^{a}$ and $x_{i}^{n}$ are the $i$ th components of the input vector before and after normalization, respectively, and $x_{i}^{\max }$ and $x_{i}^{\mathrm{min}}$ are the maximum and minimum values of all the components of the input vector before the normalization.

After the above data selection and data normalization, $250 \times 100$ samples are selected randomly to be the training samples, the rest data samples are to be a test sample. To validate the superior evaluation performance of the proposed DWMK-FSVM to evaluate the analog circuit performance online, the different methods such as LSSVR, $\varepsilon$-SVR, and the precision instrument are also carried out for the comparison purpose while the analog circuit performance evaluation is on. Meanwhile several parameters needs to be introduced before applying the three SVM algorithms. First of all, it is required to denote three parameters namely, error insensitive zone $(\varepsilon)$, penalty factor $\gamma$ and kernel specific parameters $\sigma$. Problem regarding the choice of $\varepsilon, \gamma$ and $\sigma$ was studied by several researchers $[28,29]$. The penalty factor $\gamma$ controls the smoothness or flatness of the approximation function. If we set the value $\gamma$ to be large, the objective is only to minimize the empirical risk, which makes the learning machine more complex. On the contrary, if we set the value $\gamma$ to be small, the objective is to cause the errors to be excessively tolerated yielding a learning machine with poor approximation [30]. In this study, SVM models have been constructed with $\gamma$ and $\varepsilon$ which are the empirical values given by $[31,32]$. Via some testing, the parameters $\gamma$ and $\varepsilon$ have been varied over a specific corresponding range in order to obtain better coefficient of correlation value, and the correlation value, denoted Re, is determined by (18). The kernel specific parameters $\sigma$ is restricted since the value shown in Table 1 gives the better prediction for these models. The three values for each model are shown in the following table. This study adopts $\mathrm{RBF}(16)$ where $\sigma$ is width of RBF, this is also known as kernel function. The adopted $\gamma, \varepsilon$ and $\sigma$ values for the three models are shown in Table 1 .

Consider

$$
\operatorname{Re}=\frac{\sum_{i=1}^{n}\left(D_{a i}-\bar{D}_{a}\right)\left(D_{p i}-\bar{D}_{p}\right)}{\sqrt{\sum_{i}^{n}\left(D_{a i}-\bar{D}_{a}\right)} \sqrt{\sum_{i}^{n}\left(D_{p i}-\bar{D}_{p}\right)}}
$$

where $D_{a i}$ and $D_{p i}$ are the actual and predicted values, respectively, $\bar{D}_{a}$ and $\bar{D}_{p}$ are mean of actual and predicted $D$ values corresponding to $n$ patterns.

And MSE is defined by

$$
\mathrm{MSE}=\sqrt{\frac{1}{K} \sum_{i=1}^{K}\left(Y_{i}-\overline{Y_{i}}\right)^{2}}
$$

where $Y_{i}$ is the real value, $\bar{Y}_{i}$ is the predicted value, and $K$ is a testing sample number.

To validate the superior evaluation performance of the proposed DWMK-FSVM, the other two different methods, LSSVR and $\varepsilon$-SVR, are also employed in this part. The sharp contrast about the time response of the three methods are presented in Figure 2. We take one period of testing time of LSSVR as comparison, and the other two performance evaluation methods are also employed to realize comparison in the same testing time. Via this testing comparing, we can see clearly that the testing speed is superiorly greater than the other two methods. In Figure 2, we can see that the support vector density is closely bound up the curvature. If the curvature is bigger, the support vector density is also bigger; on the contrary, while in the position of the relatively smooth, the support vector density is relatively small.

For further analysis, the maximum absolute error is presented by

$$
\mathrm{MAE}=\max \left|Y_{i}-\overline{Y_{i}}\right|
$$

From the experimental data given in Tables 2 and 3, we can find the excellent capacity of the proposed DWMK-FSVM to deal with the disturbance and fault values. And Table 4 shows the further proof that the proposed method has better evaluation performance than the other two methods.

To validate the superior evaluation performance of the proposed DWMK-FSVM, we also give out the local regression effect comparison curve of $U_{O}$ with/without fault values and disturbances under DWMK-FSVM, LSSVR, and $\varepsilon$-SVR. The sharp contrast about the time responses of the three methods are presented in Figures 2 and 3. The proposed method has the well ability to deal with the disturbance value and fault value, and the other ones do not. At the same time, the proposed method also has the same evaluation precision as the other two methods. For the same purpose, the regression effect comparison curves of $A_{u}$ with/without fault values and disturbances under DWMK-FSVM, LSSVR, and $\varepsilon$-SVR are presented in Figures 4 and 5. From the four figures, we can see that the regression effect of the proposed 


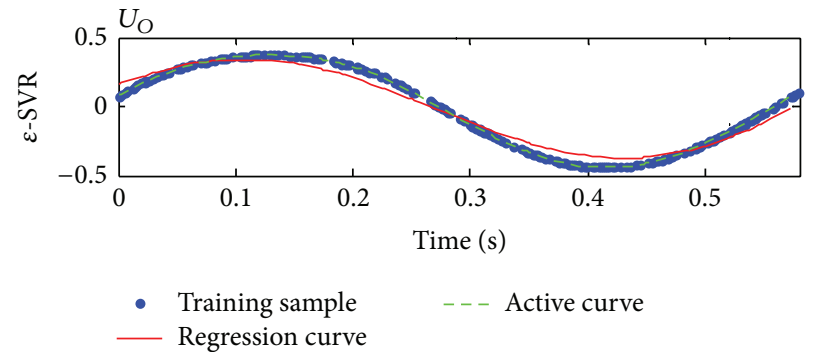

(a)

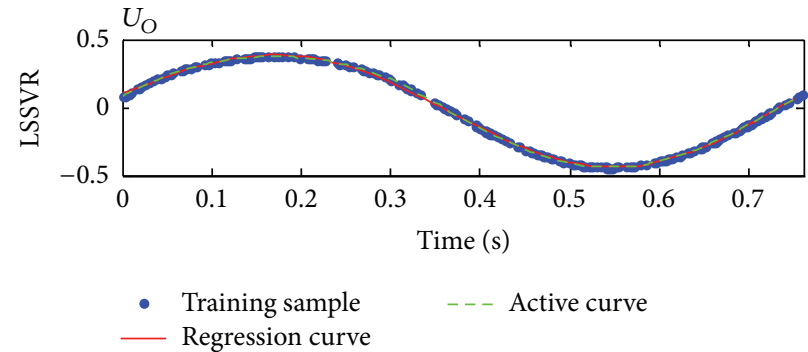

(b)

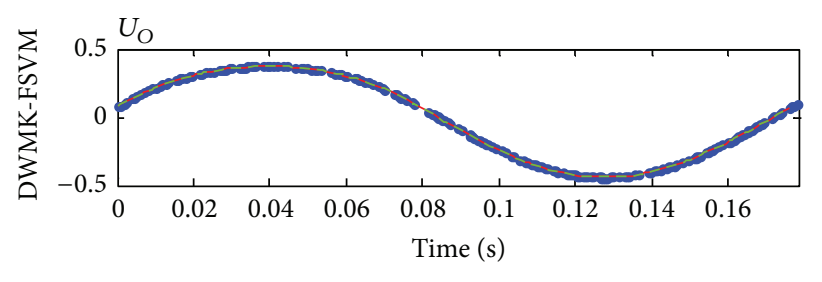

- Training sample $\quad$ - - Active curve
- Regression curve

(c)

Figure 2: The local regression effect comparison curve of $U_{O}$ without fault values and disturbances under three algorithms.

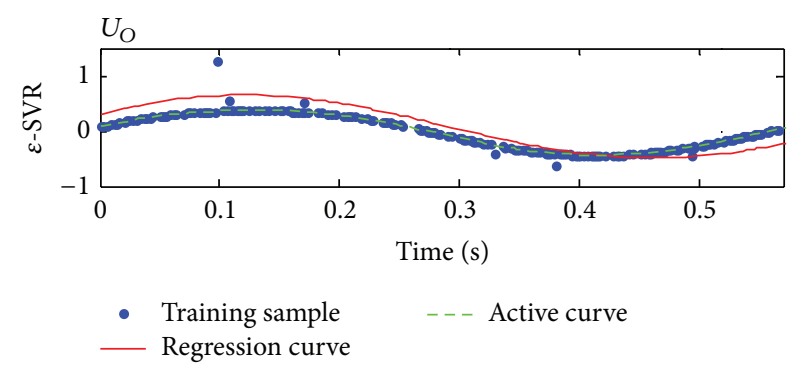

(a)

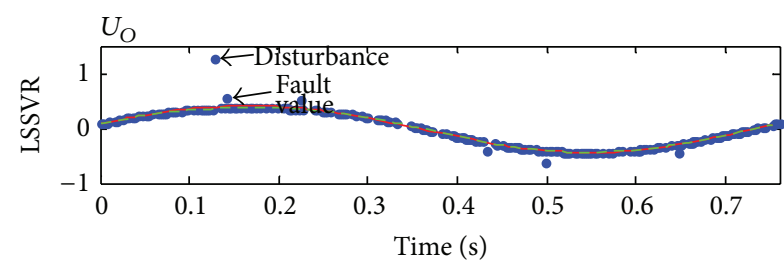

- Training sample - Regression curve

(b)

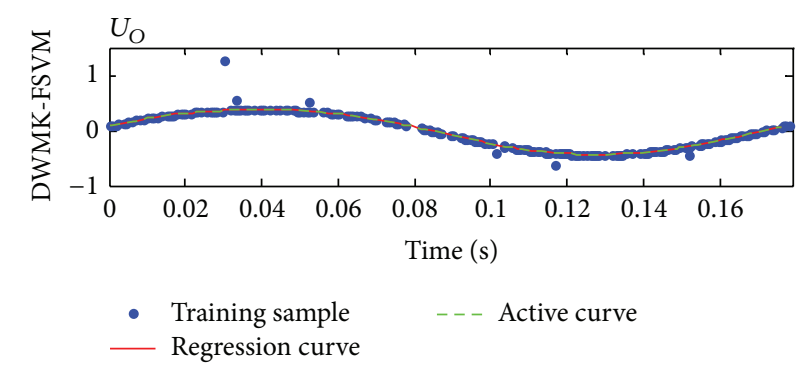

(c)

FIgURE 3: The local regression effect comparison curve of $U_{O}$ with fault values and disturbances under three algorithms.

TABLE 2: Result of data feature and comparative error of training sample.

\begin{tabular}{lcccccccrcr}
\hline \multirow{2}{*}{ TRSN } & \multirow{2}{*}{ Method } & \multicolumn{4}{c}{ Training MAE } & \multicolumn{4}{c}{ Training MSE } \\
& & $A_{u}$ & $U_{\text {om }}$ & $U_{s}$ & $U_{N}$ & $A_{u}$ & $U_{o m}$ & $U_{s}$ & $U_{N}$ \\
\hline $250 \times 100$ & DWMK-FSVM & 0.0467 & 0.0985 & 0.0894 & 0.2691 & 0.0091 & 0.0164 & 0.0094 & 0.0485 \\
$250 \times 100$ & LSSVR & 0.1164 & 0.3110 & 0.3225 & 0.6492 & 0.0780 & 0.0768 & 0.0734 & 0.2692 \\
$250 \times 100$ & $\varepsilon$-SVR & 0.1659 & 0.2217 & 0.2326 & 0.6618 & 0.2777 & 0.2576 & 0.2528 & 0.2678 \\
\hline
\end{tabular}




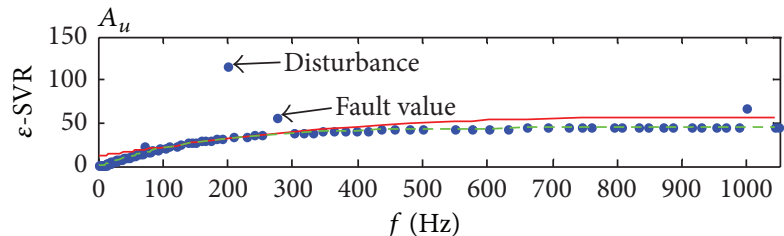

- Training sample — Regression curve

(a)

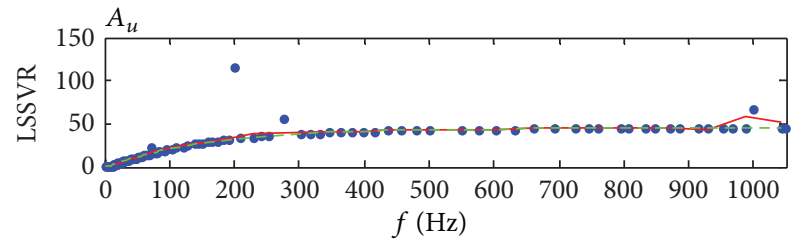

- Training sample — Regression curve

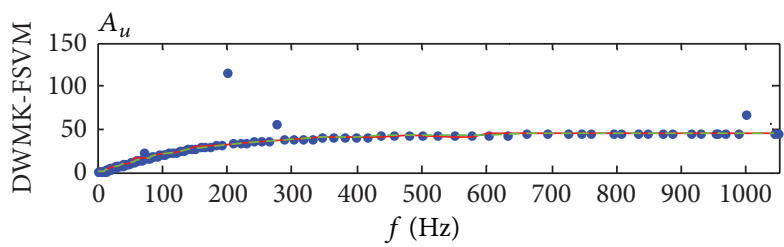

- Training sample - - - Active curve

— Regression curve

(c)

FIGURE 4: The regression effect comparison curve of $A_{u}$ with fault values and disturbances under three algorithms.

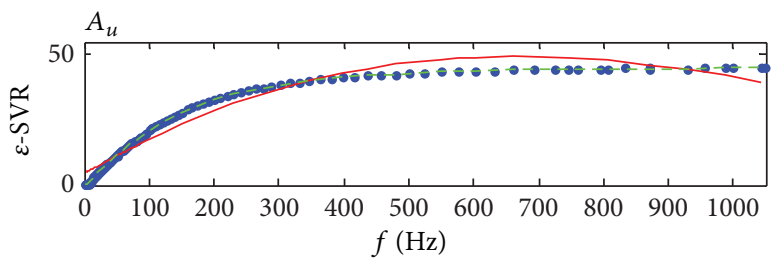

- Training sample - Regression curve

(a)

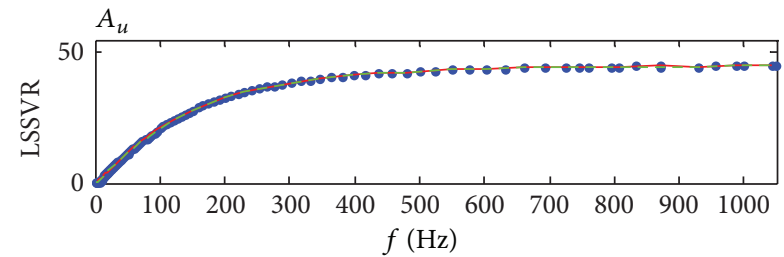

- Training sample --- Active curve - Regression curve

(b)

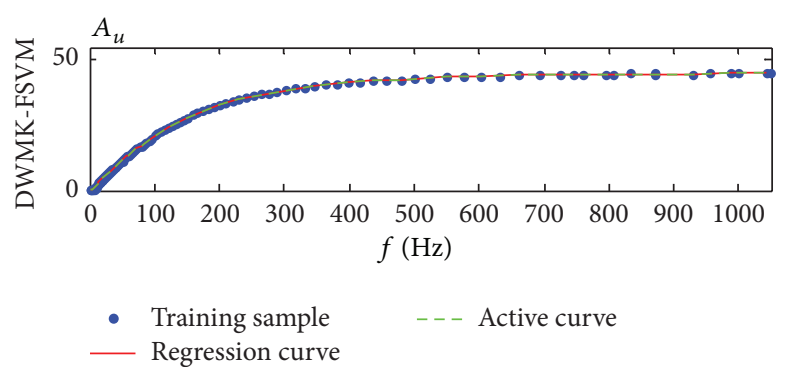

(c)

FiguRE 5: The regression effect comparison curve of $A_{u}$ without fault values and disturbances under three algorithms.

TABLE 3: Result of data feature and comparative error of testing sample.

\begin{tabular}{lcccccccrr}
\hline \multirow{2}{*}{ TSSN } & \multirow{2}{*}{ Method } & \multicolumn{4}{c}{ Test MAE } & \multicolumn{3}{c}{ Test MSE } \\
& & $A_{u}$ & $U_{\text {om }}$ & $U_{s}$ & $U_{N}$ & $A_{u}$ & $U_{\text {om }}$ & $U_{s}$ & $U_{N}$ \\
\hline $100 \times 100$ & DWMK-FSVM & 0.0667 & 0.1035 & 0.1128 & 0.1933 & 0.0201 & 0.0103 & 0.0114 & 0.0993 \\
$100 \times 100$ & LSSVR & 0.7127 & 0.3069 & 0.2369 & 0.2309 & 0.1402 & 0.1026 & 0.0765 & 0.2774 \\
$100 \times 100$ & $\mathcal{E}$-SVR & 0.7277 & 0.3478 & 0.2860 & 0.2662 & 0.2895 & 0.2719 & 0.2657 & 0.2951 \\
\hline
\end{tabular}


TABLE 4: Result of comparative assessment.

\begin{tabular}{lccccccccc}
\hline Method & $A_{u}$ & $f_{\mathrm{BW}}(\mathrm{MHz})$ & $f_{L}(\mathrm{~Hz})$ & $f_{H}(\mathrm{MHz})$ & $U_{\text {om }}(\mathrm{V})$ & $P_{\text {om }}(\mathrm{mW})$ & $U_{s}(\mathrm{mV})$ & $U_{N}(\mathrm{pV})$ & $\mathrm{CPU} / \mathrm{s}$ \\
\hline DWMK-FSVM & 46.044 & 3.8002 & 201.991 & 3.7041 & 0.4602 & 25.9489 & 10.063 & 6.2934 & 0.2056 \\
LSSVR & 51.8757 & 3.9809 & 173.7801 & 3.9811 & 0.4983 & 20.3304 & 10.5239 & 10.6661 & 0.7614 \\
$\varepsilon$-SVR & 56.5644 & 2.2906 & 336.7359 & 2.2909 & 0.6472 & 51.0814 & 16.3679 & 16.458 & 0.5796 \\
Test theory value & 46.0000 & 3.7939 & 203.0921 & 3.7941 & 0.4600 & 25.8049 & 10.0000 & 6.3840 & - \\
\hline
\end{tabular}

method is obvious better than the others, and the ability of dealing with disturbance and fault values is more remarkable than the other ones.

\section{Conclusion}

For the shortcomings of traditional analog circuit performance evaluation method for processing the wrong values, this paper presents an evaluation method based on analog circuit performance of DWMK-FSVM. Using standard SVM, combine fuzzy learning advantages with double weighted SVM to efficiently deal with the dataset that contains the wrong value. Moreover, taking into account the traditional offline evaluation strategy results in the issues that the model cannot adjust timely when the data sample of the sample group is increased or decreased; then the multi-kernel design is employed which has the ability to change the RBF width timely. And this makes the evaluation have the online processing ability. In engineering practice, considering the low-cost development, excellent accuracy evaluation, high operation speed, easy implementation and other features of evaluation methods in DWMK-FSVM, the evaluation strategy deserves development and implementation.

\section{Conflict of Interests}

The authors declare that there is no conflict of interests regarding the publication of this paper.

\section{Acknowledgments}

This present work was supported partially by the National Natural Science Foundation of China (Project no. 61304149), the Natural Science Foundation of Liaoning, China (Project no. 2013020044), and the Polish-Norwegian Research Programme (Project no. Pol-Nor/200957/47/2013). The authors highly appreciate the above financial supports.

\section{References}

[1] P. Kabisatpathy, A. Barua, and S. Sinha, Fault Diagnosis of Analog Integrated Circuits, vol. 30, Springer, New York, NY, USA, 2005.

[2] J. R. Koza, F. H. Bennett III, D. Andre, M. A. Keane, and F. Dunlap, "Automated synthesis of analog electrical circuits by means of genetic programming," IEEE Transactions on Evolutionary Computation, vol. 1, no. 2, pp. 109-128, 1997.

[3] A. E. Akadi, A. Amine, A. E. Ouardighi, and D. Aboutajdine, "A two-stage gene selection scheme utilizing MRMR filter and GA wrapper," Knowledge and Information Systems, vol. 26, no. 3, pp. 487-500, 2011.

[4] W. Song and S. C. Park, "Latent semantic analysis for vector space expansion and fuzzy logic-based genetic clustering," Knowledge and Information Systems, vol. 22, no. 3, pp. 347-369, 2010.

[5] J. Martinez-Gil and J. F. Aldana-Montes, "Evaluation of two heuristic approaches to solve the ontology meta-matching problem," Knowledge and Information Systems, vol. 26, no. 2, pp. 225-247, 2011.

[6] C.-W. Chen, P.-C. Chen, and W.-L. Chiang, "Modified intelligent genetic algorithm-based adaptive neural network control for uncertain structural systems," Journal of Vibration and Control, vol. 19, no. 9, pp. 1333-1347, 2013.

[7] S. K. Shevade, S. S. Keerthi, C. Bhattacharyya, and K. R. K. Murthy, "Improvements to the SMO algorithm for SVM regression," IEEE Transactions on Neural Networks, vol. 11, no. 5, pp. 1188-1193, 2000.

[8] B. Xiao, Q. L. Hu, and Y. M. Zhang, "Adaptive sliding mode fault tolerant attitude tracking control for flexible spacecraft under actuator saturation," IEEE Transactions on Control Systems Technology, vol. 20, no. 6, pp. 1605-1612, 2011.

[9] B. Xiao, Q. L. Hu, and G. Ma, "Adaptive sliding mode backstepping control for attitude tracking of flexible spacecraft under input saturation and singularity," Proceedings of the Institution of Mechanical Engineers G, vol. 224, no. 2, pp. 199-214, 2010.

[10] S. Sra, S. Nowozin, and S. J. Wright, Optimization for Machine Learning, 2011.

[11] S. Abdulla and M. Tokhi, "Fuzzy logic based FES driven cycling by stimulating single muscle group," in Converging Clinical and Engineering Research on Neurorehabilitation, pp. 173-182, Springer, New York, NY, USA, 2013.

[12] S. Yin, H. Luo, and S. Ding, "Real-time implementation of faulttolerant control systems with performance optimization," IEEE Transactions on Industrial Electronics, vol. 61, no. 5, pp. 24022411, 2013.

[13] X. Zhao, L. Zhang, P. Shi, and H. Karimi, "Robust control of continuous-time systems with state-dependent uncertainties and its application to electronic circuits," IEEE Transactions on Industrial Electronics, 2013.

[14] X. Zhao, L. Zhang, P. Shi, and M. Liu, "Stability of switched positive linear systems with average dwell time switching," Automatica, vol. 48, no. 6, pp. 1132-1137, 2012.

[15] X. Zhao, X. Liu, S. Yin, and H. Li, "Improved results on stability of continuous-time switched positive linear systems," Automatica, 2013.

[16] X. Zhao, L. Zhang, P. Shi, and M. Liu, "Stability and stabilization of switched linear systems with mode-dependent average dwell time," IEEE Transactions on Automatic Control, vol. 57, no. 7, pp. 1809-1815, 2012.

[17] S. Yin, S. X. Ding, A. H. A. Sari, and H. Hao, "Data-driven monitoring for stochastic systems and its application on batch 
process," International Journal of Systems Science, vol. 44, no. 7, pp. 1366-1376, 2013.

[18] S. Yin, S. X. Ding, A. Haghani, H. Hao, and P. Zhang, "A comparison study of basic data-driven fault diagnosis and process monitoring methods on the benchmark Tennessee Eastman process," Journal of Process Control, vol. 22, no. 9, pp. 1567-1581, 2012.

[19] S. Yin, X. Yang, and H. R. Karimi, "Data-driven adaptive observer for fault diagnosis," Mathematical Problems in Engineering, vol. 2012, Article ID 832836, 21 pages, 2012.

[20] A. Zhang and Z. Yu, "Research on amplifier performance evaluation based on support vector regression machine," Chinese Journal of Scientific Instrument, vol. 29, no. 3, pp. 618-622, 2008.

[21] K.-H. Park, Z. Bien, and D.-H. Hwang, "A study on the robustness of a PID-type iterative learning controller against initial state error," International Journal of Systems Science, vol. 30, no. 1, pp. 49-59, 1999.

[22] P. R. Ouyang, W. J. Zhang, and M. M. Gupta, "An adaptive switching learning control method for trajectory tracking of robot manipulators," Mechatronics, vol. 16, no. 1, pp. 51-61, 2006.

[23] S. Kawamura, F. Miyazaki, and S. Arimoto, "Realization of robot motion based on a learning method," IEEE Transactions on Systems, Man and Cybernetics, vol. 18, no. 1, pp. 126-134, 1988.

[24] C.-F. Lin and S.-D. Wang, "Fuzzy support vector machines," IEEE Transactions on Neural Networks, vol. 13, no. 2, pp. 464471, 2002.

[25] M. Hall, E. Frank, G. Holmes, B. Pfahringer, P. Reutemann, and I. H. Witten, "The WEKA data mining software: an update," ACM SIGKDD Explorations Newsletter, vol. 11, no. 1, pp. 10-18, 2009.

[26] D. E. Lee, J.-H. Song, S.-O. Song, and E. S. Yoon, "Weighted support vector machine for quality estimation in the polymerization process," Industrial and Engineering Chemistry Research, vol. 44, no. 7, pp. 2101-2105, 2005.

[27] A. Zhang, C. Chen, and H. R. Karimi, "A new adaptive LSSVR with online multikernel RBF tuning to evaluate analog circuit performance," Abstract and Applied Analysis, vol. 2013, Article ID 231735, 7 pages, 2013.

[28] V. N. Vapnik, The Nature of Statistical Learning Theory, Statistics for Engineering and Information Science, Springer, New York, NY, USA, 2000.

[29] S. R. Gunn, "Support vector machines for classification and regression," ISIS Technical Report 14, 1998.

[30] S. Rajasekaran, S. Gayathri, and T.-L. Lee, "Support vector regression methodology for storm surge predictions," Ocean Engineering, vol. 35, no. 16, pp. 1578-1587, 2008.

[31] P.-S. Yu, S.-T. Chen, and I.-F. Chang, "Support vector regression for real-time flood stage forecasting," Journal of Hydrology, vol. 328, no. 3-4, pp. 704-716, 2006.

[32] Z. Liang and Y. Li, "Incremental support vector machine learning in the primal and applications," Neurocomputing, vol. 72, no. 10-12, pp. 2249-2258, 2009. 


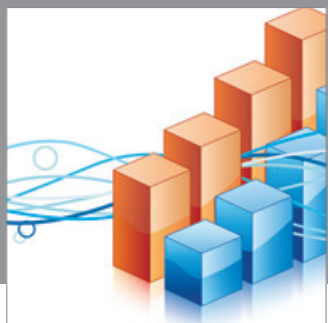

Advances in

Operations Research

mansans

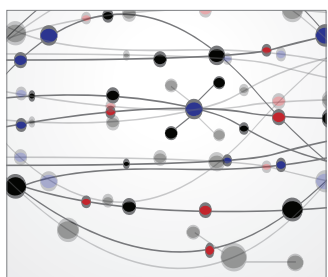

The Scientific World Journal
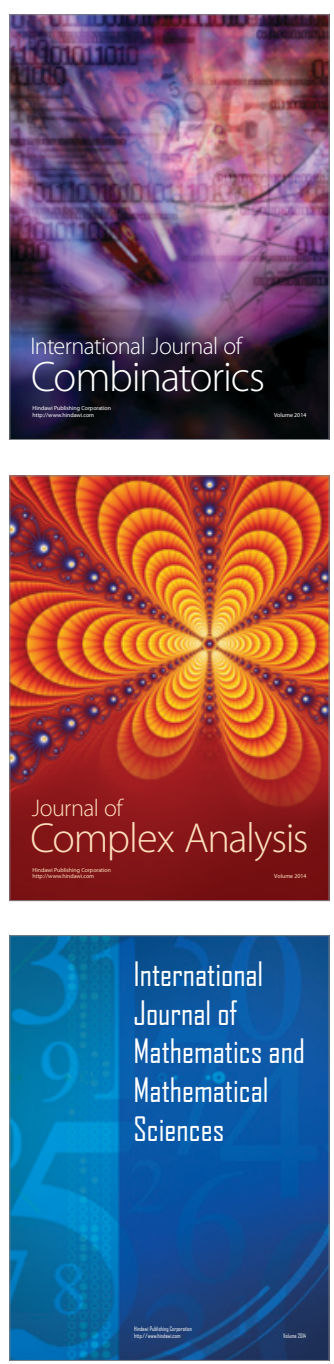
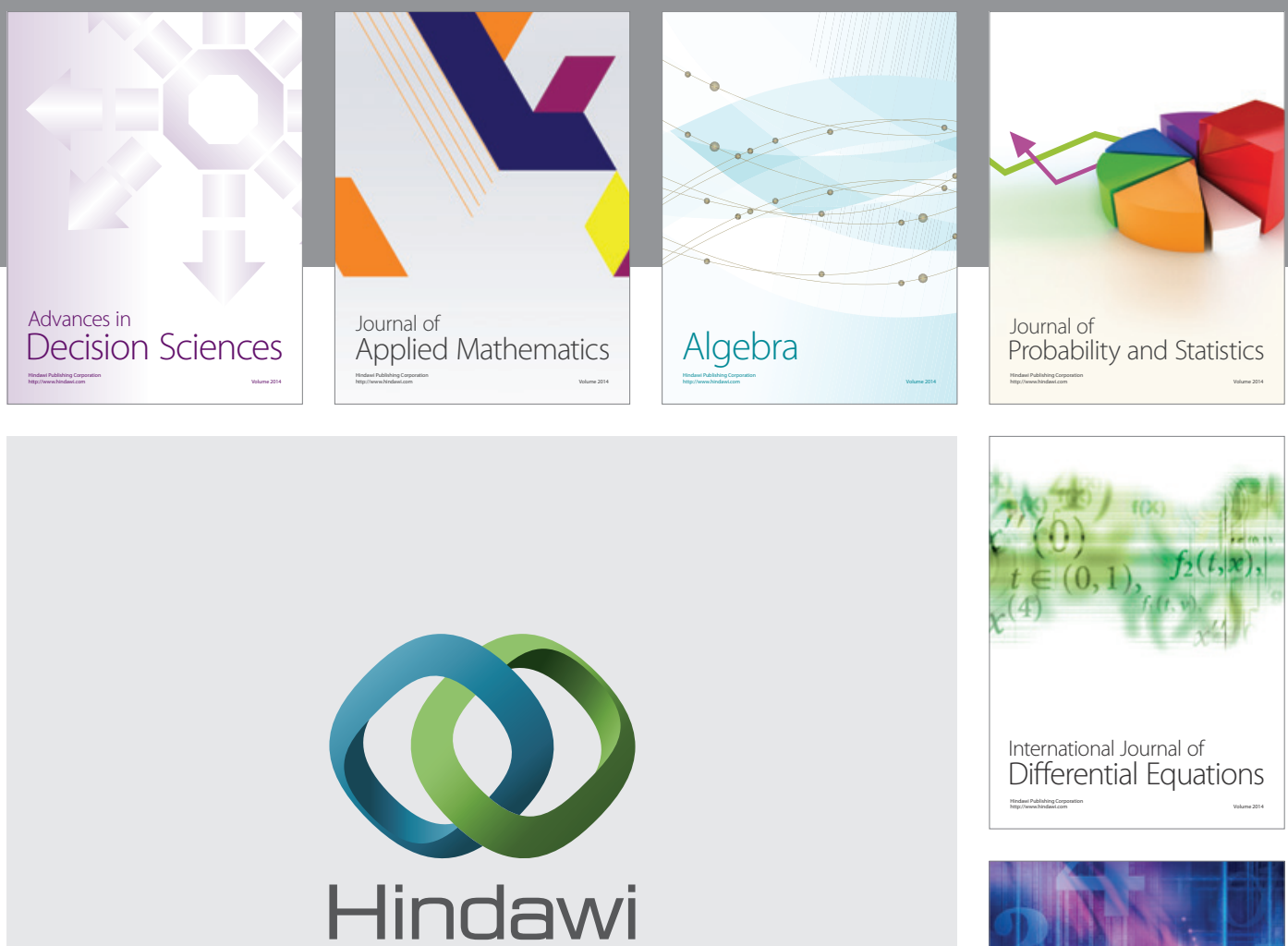

Submit your manuscripts at http://www.hindawi.com
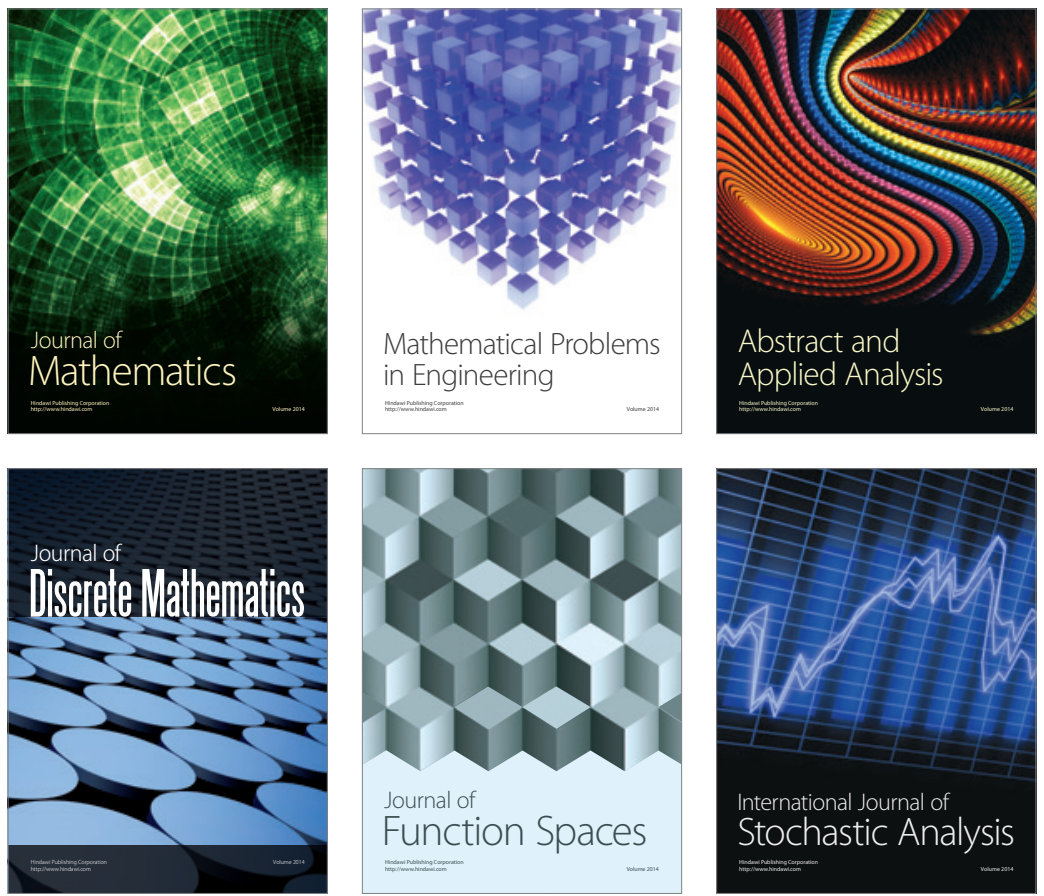

Journal of

Function Spaces

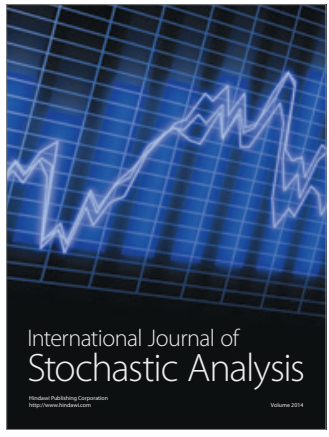

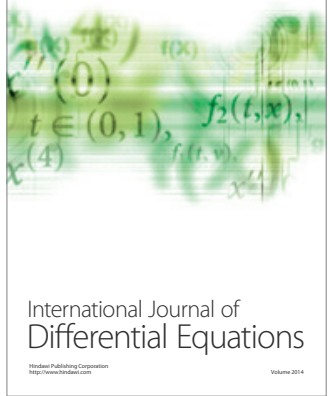
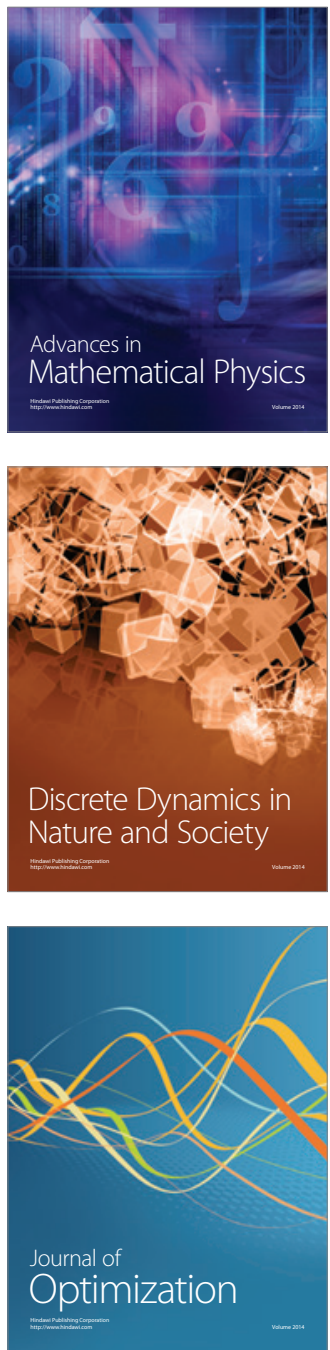